\title{
Machine Learning for Robotics
}

\author{
Pieter Abbeel \\ University of California, Berkeley, USA \\ pabbeel@cs.berkeley.edu \\ http://www.cs.berkeley.edu/ pabbeel/
}

\begin{abstract}
Robots are typically far less capable in autonomous mode than in teleoperated mode. The few exceptions tend to stem from long days (and more often weeks, or even years) of expert engineering for a specific robot and its operating environment. Current control methodology is quite slow and labor intensive. I believe advances in machine learning have the potential to revolutionize robotics. In this talk, I will present new machine learning techniques we have developed that are tailored to robotics. I will describe in depth "Apprenticeship learning", a new approach to high-performance robot control based on learning for control from ensembles of expert human demonstrations. Our initial work in apprenticeship learning has enabled the most advanced helicopter aerobatics to-date, including maneuvers such as chaos, tic-tocs, and auto-rotation landings which only exceptional expert human pilots can fly. Our most recent work in apprenticeship learning is providing traction on learning to perform challenging robotic manipulation tasks, such as knot-tying. I will also briefly highlight three other machine learning for robotics developments: Inverse reinforcement learning and its application to quadruped locomotion, Safe exploration in reinforcement learning which enables robots to learn on their own, and Learning for perception with application to robotic laundry.
\end{abstract}

\section{Bio}

Pieter Abbeel received a BS/MS in Electrical Engineering from KU Leuven (Belgium) and received his Ph.D. degree in Computer Science from Stanford University in 2008. He joined the faculty at UC Berkeley in Fall 2008, with an appointment in the Department of Electrical Engineering and Computer Sciences. He has won various awards, including best paper awards at ICML and ICRA, the Sloan Fellowship, the Air Force Office of Scientific Research Young Investigator Program (AFOSR-YIP) award, the Okawa Foundation award, the 2011s TR35, and the IEEE Robotics and Automation Society (RAS) Early Career Award. He has developed apprenticeship learning algorithms which have enabled advanced helicopter aerobatics, including maneuvers such as tic-tocs, chaos and auto-rotation, which only exceptional human pilots can perform. His group has also enabled the first end-to-end completion of reliably picking up a crumpled laundry article and folding it. His work has been featured in many popular press outlets, including BBC, New York Times, MIT Technology Review, Discovery Channel, SmartPlanet and Wired. His current research focuses on robotics and machine learning with a particular focus on challenges in personal robotics, surgical robotics and connectomics. 